\title{
Evaluating a preoperative protocol that includes magnetic resonance imaging for lymph node metastasis in the Cholangiocarcinoma Screening and Care Program (CASCAP) in Thailand
}

Metha Songthamwat ${ }^{1}$, Nittaya Chamadol ${ }^{2,3,4,5}$, Narong Khuntikeo ${ }^{3,4,5,6}$, Jadsada Thinkhamrop ${ }^{7}$, Supinda Koonmee ${ }^{8}$, Nathaphop Chaichaya ${ }^{9}$, Jeffrey Bethony ${ }^{10}$ and Bandit Thinkhamrop ${ }^{1,3,9^{*}}$ (D)

\begin{abstract}
Background: Treatment planning especially liver resection in cholangiocarcinoma (CCA) depends on the extension of tumor and lymph node metastasis which is included as a key criterion for operability. Magnetic resonance imaging (MRI) offers a rapid and powerful tool for the detection of lymph node metastasis (LNM) and in the current manuscript is assessed as a critical tool in the preoperative protocol for liver resection for treatment of CCA. However, the accuracy of MRI to detect LNM from CCA had yet to be comprehensively evaluated.

Methods: The accuracy of MRI to detect LNM was assessed in a cohort of individuals with CCA from the Cholangiocarcinoma Screening and Care Program (CASCAP), a screening program designed to reduce CCA in Northeastern Thailand by community-based ultrasound (US) for CCA. CCA-positive individuals are referred to one of the nine tertiary centers in the study to undergo a preoperative protocol that included enhanced imaging by MRI. Additionally, these individuals also underwent lymph node biopsies for histological confirmation of LNM (the "gold standard") to determine the accuracy of the MRI results.

Results: MRI accurately detected the presence or absence of LNM in only 29 out of the 51 CCA cases (56.9\%, 95\% Cl 42.2-70.7), resulting in a sensitivity of $57.1 \%$ (95\% Cl 34.0-78.2) and specificity of 56.7\% (95\% Cl 37.4-74.5), with positive and negative predictive values of $48.0 \%(95 \% \mathrm{Cl} 27.8-68.7)$ and $65.4 \%(95 \% \mathrm{Cl} 44.3-82.8)$, respectively. The positive likelihood ratio was 1.32 (95\% Cl 0.76-2.29), and the negative likelihood ratio was 0.76 (95\% Cl 0.42-1.36).

Conclusions: MRI showed limited sensitivity and a poor positive predictive value for the diagnosis of LNM for CCA, which is of particular concern in this resource-limited setting, where simpler detection methods could be utilized that are more cost-effective in this region of Thailand. Therefore, the inclusion of MRI, a costly imaging method, should be reconsidered as part of protocol for treatment planning of CCA, given the number of false positives, especially as it is critical in determining the operability for CCA subjects.
\end{abstract}

Keywords: Cholangiocarcinoma, Magnetic resonance imaging, Lymph node metastasis, Accuracy, Preoperative protocol

\footnotetext{
* Correspondence: bandit@kku.ac.th

${ }^{1}$ Faculty of Public Health, Khon Kaen University, Khon Kaen, Thailand

${ }^{3}$ Cholangiocarcinoma Screening and Care Program (CASCAP), Khon Kaen

University, Khon Kaen, Thailand

Full list of author information is available at the end of the article
} 


\section{Background}

Cholangiocarcinoma (CCA) is a primary hepatic malignancy that arises along the intrahepatic and extrahepatic bile ducts. It is the second most common liver cancer globally [1] and the most common liver cancer in the resource-limited setting of the northeastern provinces of Thailand [2], where its high incidence is due to risk factors unique to this tropical disease, i.e., the consumption of raw or undercooked cyprinoid fish, which are the intermediate hosts for the food-borne trematode Opisthorchis viverrini $(\mathrm{Ov})$, one of the few carcinogenic pathogens [3]. When detected early, CCA is amenable to liver resection, which substantially improves survival $[4,5]$, with the median survival time of liver resection patients at 23 months compared with less than 8 months in inoperable patients [5]. However, due to the asymptomatic or non-specific nature of the symptoms associated with CCA during its early stages, this cancer is often detected at an advanced stage, with a concomitantly poor prognosis and dismal survival rate [5]. The Cholangiocarcinoma Screening and Care Program (CASCAP) was instituted in Northeast Thailand with the objective of conducting community-based ultrasound (US) screening programs for the early diagnosis of CCA, making liver resection a viable post-diagnostic option for the treatment of CCA [6].

As the objective of CASCAP is to increase survival from CCA by aggressively screening for this cancer by mobile US units in Ov-endemic areas, a critical component of this endeavor was to establish a preoperative evaluation protocol for liver resection for CCA, which would include factors such as (1) tumor infiltration beyond the second order bile duct branches, (2) tumor invasion of major vessels such as main hepatic artery or portal vein, and (3) the extent of lymph node metastasis (LNM) [7-15]. Due to its location in the hepatoduodenal ligament, CCA quickly metastasizes outside the liver via the lymph node system or perineural invasion $[16,17]$, even during the early stages of this bile duct cancer. While several methods are available for detecting LNM, CASCAP attempted magnetic resonance imaging (MRI) to scan for LNM as part of a preoperative evaluation protocol for CCA due to its speed and "accuracy" in other cancer contexts [18]. However, the accuracy of MRI for depicting LNM for CCA has to be studied for each individual cancer, and it has been poorly studied of CCA [19-21]. The assessment of LNM by MRI for CCA is seldom performed due to limited access to tertiary care centers in this resourcepoor setting. Herein, we investigated the diagnostic performance of MRI for the detection of LNM, as a part of the preoperative evaluation protocol for CCA, compared to the "gold standard" of pathological confirmation of individuals suspected of CCA by lymph node resection.

\section{Methods}

Study setting: community-based risk stratification and ultrasonography

This study was based on data from CASCAP, which is a combined community and hospital-based cohort study conducted in Ov-endemic areas in Northeastern Thailand (www.cascap.in.th) and nine tertiary care hospitals in this same region. The CASCAP study is comprised of two cohorts. The first cohort is the "screening cohort," which includes individuals who were risk stratified using criteria consisting of (1) their residence in Northeastern Thailand, (2) age of 40 years or older (inclusive), (3) previous reported infection with the carcinogenic liver fluke Ov, (4) previously reported treatment for Ov infection with praziquantel, and (5) self-reported consumption of raw or undercooked freshwater fish, the second intermediate host of Ov. Individuals considered at risk for Ov-induced CCA underwent liver US as detailed in Khuntikeo et al. [6]. The second cohort is a conventional cancer registry, where patients were suspected of CCA during a routine US from one of the nine participating hospitals.

\section{Magnetic resonance cholangiopancreatography}

Individuals included in the present study included those enrolled in CASCAP who were suspected of CCA by US or clinical symptoms and had a confirmatory magnetic resonance cholangiopancreatography (MRCP) or computerized tomography (CT) scanning at one of nine tertiary care centers. The MRI used a 1.5-3.0 T system. Two different MRCP techniques were applied: a single-shot rapid acquisition with relaxation enhancement (RARE) and a multislice half-Fourier acquisition single-shot turbo spin echo (HASTE). The slabs of the single-shot RARE sequence were obtained on various planes (e.g., coronal, axial, and oblique) to allow for the optimal visualization of the bile ducts. The number of thick-slab acquisitions ranged from seven to nine per patient. Next, multislice HASTE images were obtained in the coronal and oblique planes. Each examination was performed during a single breath-hold.

The imaging parameters for the single-shot RARE sequence were as follows: repetition time of $\infty$, effective echo time of $1200 \mathrm{~ms}$, echo train length of 240, flip angle of $150^{\circ}$, slab thickness of 70 to $90 \mathrm{~mm}$, field of view of 300 to $340 \mathrm{~mm}$, matrix of $240 \times 256$, and an acquisition time of $2.32 \mathrm{~s}$. The imaging parameters for the multislice HASTE sequence were as follows: repetition time of $\infty$, effective echo time of $95 \mathrm{~ms}$, echo train length of 128; flip angle of $150^{\circ}$, section thickness of $4 \mathrm{~mm}$ without a gap and 13 to 15 slices (range of coverage $52-60 \mathrm{~mm}$ ), field of view of 300 to $340 \mathrm{~mm}$, matrix of $240 \times 256$, and an acquisition time of 18 to $20 \mathrm{~s}$. Fat saturation was used to reduce a strong fat signal during image acquisition. The total acquisition time for all of the imaging steps in the MR imaging were less than $15 \mathrm{~min}$. 


\section{LNM determination}

The determination of LNM by MRI was made by a consensus between two board-certified radiologists using the following morphological criteria: nodal size greater than $10 \mathrm{~mm}$ in short axis diameter, central necrosis, and inhomogeneous enhancement following intravenous contrast medium injection [22-24]. In equivocal cases, a gastrointestinal radiologist was also consulted. The determination for surgery was attained by a consensus between the surgeon and the patient, after the counseling process was completed.

\section{Gold standard pathological evaluation of LNM}

In the case when a surgical treatment occurred, the operation types and plans were discussed with the doctors before the procedure. The time interval between MRI scan and operation was about 1 month. Lymph node dissections were performed in case of the presence of an enlarged or abnormal consistency of the lymph nodes. These dissected lymph nodes were sent for pathological examination. The diagnosis of pathological LNM was done by two boardcertified pathologists. The procedures included each pathologist performing a gross and microscopic examination of the tissue specimen separately with the pathological diagnosis and lymph node metastasis made by consensus. In case of differences in the diagnosis between the two pathologists, immunohistochemistry strains were used to determine a consensus between both pathologists.

\section{Data analysis}

The descriptive statistics of patients and the type of tumor were presented as mean and standard deviations, such as age. Categorical variables were presented as numbers and percentages. A comparison between LNM diagnosis using an MRI scan and pathological diagnosis included the following parameters: sensitivity, specificity, positive predictive values (PPV), negative predictive values (NPV), false positive rates, and false negative rates. They were calculated along with their $95 \%$ confidence intervals $(95 \% \mathrm{CI})$. These diagnostic parameters were presented as either the overall CCA or CCA stratified into intrahepatic, perihilar, and distal type based on the American Joint Committee on Cancer (AJCC) criteria. All statistical analyses were conducted using Stata13 (Stata Corp, College Station, TX, USA). Attached data sets 1-3 were used for all analysis (Additional files 1, 2 and 3).

\section{Ethical approval}

This study was conducted according to the principles of Good Clinical Practice (Chapter 2 of the International Conference of Harmonized Tripartite Guideline for Good Clinical Practice), the Declaration of Helsinki, and the national laws and regulations about clinical studies. CASCAP was approved by Khon Kaen University Ethics Committee
(HE551404) for Human Research and received written informed consent from all patient participants.

\section{Results}

A total of 433 individuals suspected of CCA by US were sent for MRI scanning, with a follow-up confirmation of 255 cases of CCA (58.9\%). There were 133 (52.2\%) individuals with intrahepatic CCA, 112 (43.9\%) with perihilar CCA, and 10 (3.92\%) with distal CCA. In the CCA group confirmed by MRI scans, 248 (97.3\%) subjects also had radiographic lymph node findings, with 125 (50.4\%) showing LNM. Of these 255 MRI-confirmed CCA subjects, 130 received surgical treatments with pathologically confirmed CCA. The 51 underwent lymph node dissection, with only 21 (41.2\%) having confirmed LNM based on the conventional pathological diagnosis (Fig. 1).

Of the 51 individuals who underwent lymph node dissections, the mean age was found to be 61.5 years (SD 10.3), with 33 out of 51 (64.7\%) being males. Of these individuals, $35.3 \%$ had intrahepatic, $60.8 \%$ perihilar, and $3.9 \%$ distal CCA (Table 1).

The MRI scan accurately detected the presence or absence of LNM in 29 out of 51 CCA cases $(56.9 \%, 95 \%$ CI $42.2-70.7)$, resulting in a sensitivity of $57.1 \%(95 \% \mathrm{CI}$ $34.0-78.2)$ and a specificity of $56.7 \%$ (95\% CI 37.4-74.5), with a PPV and a NPV of $48.0 \%$ (95\% CI 27.8-68.7) and $65.4 \%$ (95\% CI 44.3-82.8), respectively. The positive likelihood ratio was 1.32 (95\% CI $0.76-2.29)$, and the negative likelihood ratio was $0.76(95 \%$ CI $0.42-1.36)$ (Tables 2 and 3).

For intrahepatic CCA, the MRI scan had a sensitivity of $50.0 \%$ (95\% CI $15.7-84.3$ ) and a specificity of $88.9 \%$ (95\% CI 51.8-99.7), with a PPV and a NPV of $80.0 \%$ (95\% CI 28.4-99.5) and 66.7\% (95\% CI 34.9-90.1), respectively. The positive likelihood ratio was 4.50 (95\% CI 0.63-32.4), and the negative likelihood ratio was 0.56 (95\% CI $0.27-$ 1.17) (Table 3). In the case of perihilar CCA, the MRI scan had a sensitivity of $61.5 \%$ (95\% CI 31.6-86.1) and a specificity of $38.9 \%$ (95\% CI 17.3-64.3), with a PPV and NPV of $42.1 \%$ (95\% CI 20.3-66.5) and 58.3\% (95\% CI $27.7-$ $84.8)$, respectively. The positive likelihood ratio was 1.01 (95\% CI $0.57-1.77$ ), and the negative likelihood ratio was 0.99 (95\% CI 0.40-2.43) (Table 3).

\section{Discussion}

The age-adjusted incidence rates for CCA in Northeastern Thailand are 89.2 and 35.5 per 100,000 for males and females, respectively, which are among the highest in the world. The high incidence of this relatively rare cancer in Northeast Thailand is due to the dietary habits of the inhabitants of the region, who consume raw or undercooked cyprinoid fish, the intermediate host of the food-borne pathogen Opisthorchis viverrini. CCA tends to present late, often going undetected until an advanced stage, when 


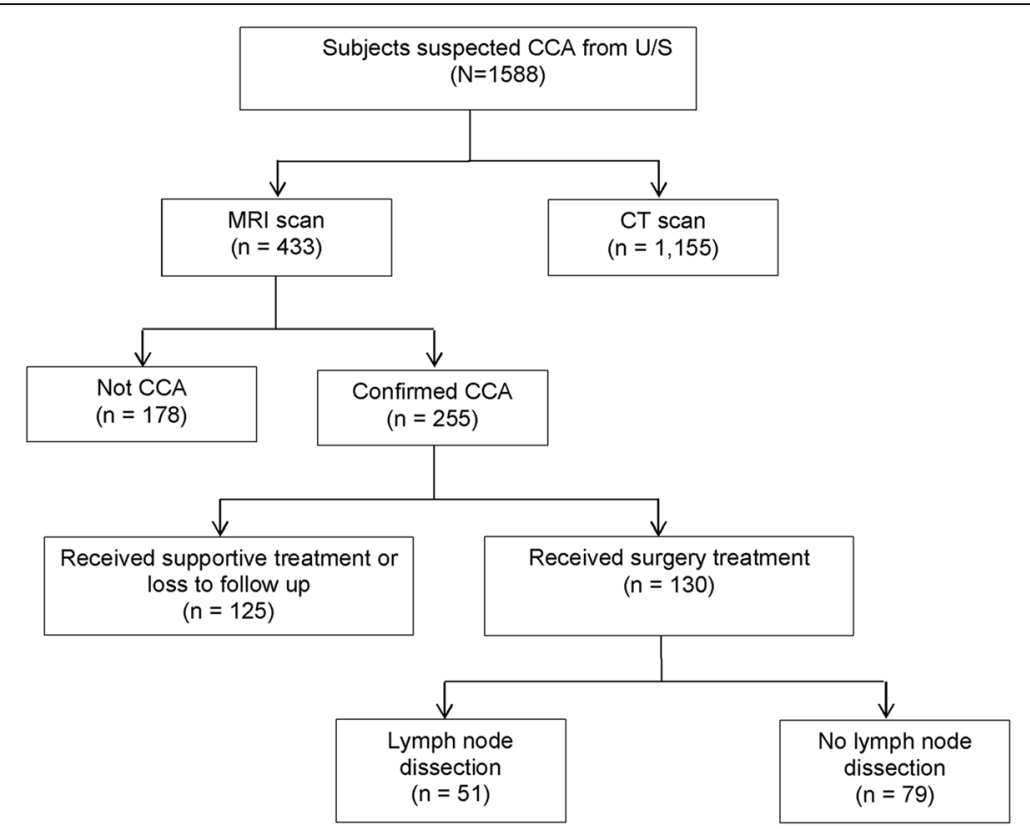

Fig. 1 Flow of subjects in the Cholangiocarcinoma Screening and Care Program (CASCAP). Abbreviations: CCA cholangiocarcinoma, U/S ultrasonography, CT computerized tomography, MRI magnetic resonance imaging

overall survival is less than 12 months [4, 25-29]. Currently, the most successful treatment for CCA is radical liver resection at an early stage of the disease and in the absence of LNM. With its location in the hepatoduodenal ligament, CCA tumors tend to metastasize outside the liver either via the lymph node system or via perineural invasion [16, 17]. Health disparities in this region of Thailand exacerbate this poor prognosis, as there are limited early cancer screenings and minimal post-diagnostic treatment. CASCAP was instituted in Northeastern Thailand to resolve these health disparities associated with CCA by conducting community-based US screening to detect early CCA.

The development of a preoperative protocol for CCA is central to the strategy of CASCAP. Currently, the preoperative protocol for CCA includes an (1) extension of

Table 1 Characteristics of patients and type of tumors presented as number and percentage unless specified otherwise

\begin{tabular}{llll}
\hline & & Subjects & Percent \\
\hline Mean age \pm SD & & $61.5 \pm 10.3$ years & \\
& & & \\
& Male & 33 & 64.7 \\
& Female & 18 & 35.3 \\
Type of tumor & & & \\
& Intrahepatic & 18 & 35.3 \\
& Perihilar & 31 & 60.8 \\
& Distal & 2 & 3.9 \\
\hline SD standard deviation & & &
\end{tabular}

the cancer to the hepatic artery or portal vein and (2) extension of lymph node metastasis (LNM) [7-15]. CCA cases that underwent liver resection in the presence of pathologically confirmed LNM were reported to have a zero 5-year survival rate [30-33], whereas CCA cases that underwent liver resection in the absence of LNM were reported to have a $35-72 \%$ 5-year survival rate [33-36]. However, the specific outcome of liver MRI on the surgical management of CCA has yet to be systematically evaluated. While several studies have estimated the diagnostic accuracy of MRI as part of the preoperative evaluation of bile duct involvement and vascular involvement $[19,20,37]$, our study is the first to have determined the accuracy of MRI in the preoperative evaluation of LNM in CCA.

In the current study, MRIs were observed to have limited utility for preoperative evaluation for CCA compared to the "gold standard" of pathological confirmation of LNM. An especially concerning finding was the high false

Table 2 Comparing the result of magnetic resonance imaging and pathological diagnosis (subjects)

\begin{tabular}{lllll}
\hline & & \multicolumn{2}{l}{ Lymph node pathology } & Total \\
\cline { 2 - 4 } & & Metastasis & Non-metastasis & \\
\hline $\begin{array}{lllll}\text { Magnetic resonance } \\
\text { imaging scan }\end{array}$ & $\begin{array}{l}\text { Nodal } \\
\text { metastasis }\end{array}$ & 12 & 13 & 25 \\
& $\begin{array}{l}\text { Non-nodal } \\
\text { metastasis }\end{array}$ & 9 & 17 & 26 \\
& $\begin{array}{l}\text { Total } \\
\text { Totann }\end{array}$ & 21 & 30 & 51 \\
\hline
\end{tabular}


Table 3 Diagnostic performance of magnetic resonance imaging in diagnosis of lymph node metastasis in different types of cholangiocarcinoma

\begin{tabular}{|c|c|c|c|c|c|c|}
\hline \multirow[b]{2}{*}{ CCA } & \multicolumn{2}{|l|}{ Diagnostic } & \multicolumn{2}{|l|}{ Predictive value } & \multicolumn{2}{|l|}{ Likelihood ratio } \\
\hline & Sensitivity $(95 \% \mathrm{Cl})$ & Specificity (95\% CI) & Positive $(95 \% \mathrm{Cl})$ & Negative $(95 \% \mathrm{Cl})$ & Positive $(95 \% \mathrm{Cl})$ & Negative (95\% Cl) \\
\hline \multirow[t]{2}{*}{ Intrahepatic } & 50.0 & 88.9 & 80.0 & 66.7 & 4.50 & 0.56 \\
\hline & $(23.0-72.2)$ & $(51.8-99.7)$ & $(28.4-99.5)$ & $(34.9-90.1)$ & $(0.63-32.9)$ & $(0.27-1.17)$ \\
\hline \multirow[t]{2}{*}{ Perihilar } & 61.5 & 38.9 & 42.1 & 58.3 & 1.01 & 0.99 \\
\hline & $(31.6-86.1)$ & $(17.3-64.3)$ & $(20.3-66.5)$ & $(27.7-84.8)$ & $(0.57-1.77)$ & $(0.40-2.43)$ \\
\hline \multirow[t]{2}{*}{ Overall } & 57.1 & 56.7 & 48.0 & 65.4 & 1.32 & 0.76 \\
\hline & (34.0-78.2) & (37.4-74.5) & $(27.8-68.7)$ & (44.3-82.8) & $(0.76-2.29)$ & $(0.42-1.36)$ \\
\hline
\end{tabular}

CCA cholangiocarcinoma, $\mathrm{Cl}$ confidence interval

positive rate of MRIs for CCA LNM, which would exclude individuals otherwise eligible for surgery. We quantified the sensitivity of MRI scans in determining LNM for CCA at $57.1 \%$, which was far lower than other preoperative resectability criteria such as the bile duct and vascular involvement [19, 20, 37]. A factor that may limit the accuracy of MRI scans in the assessment of CCA LNM is the reliance on nodal size in the axial short axis because of the inability to detect microscopic metastasis in normal-size nodes and to distinguish benign enlargement from malignant lymph nodes [12]. Our findings are similar to a recent study that found the survival rate of CCA with preoperative lymph node enlargement improved after hepatectomy [38]. We also agree with a study that recommended routine lymphadenectomy in all cases of hepatectomy [36]. An important limitation of this study is that MRI scanning was carried out at nine different tertiary care centers, using different machines, techniques, and radiologists. This indicates the possibility of variation in the data collection. Second, the lymph node pathology, which is the gold standard of this study, was determined in only some surgical subjects: not all MRI scans confirmed CCA diagnosis. The site and number of lymph nodes from MRI and pathological diagnosis also might be different. This might have an effect on our results. Finally, the time between an MRI scan and surgical operation might have affected the progression of the disease, as it may affect the period during which LNM may occur.

Liver resection has long been shown to increase survival from CCA, though it is highly dependent on LNM. Herein, we utilized the unique early screening program and a large sample size (CASCAP) in Northeastern Thailand to determine the quality of data for MRI scanning to determine LNM in the preoperative evaluation for CCA. We found that MRI scanning for LNM had limited sensitivity, with a low positive predictive value, and a high number of false positives, which would define eligible patients as inoperable. The evaluation of the nodal metastasis based on this radiological imaging modality has significant clinical implications for the surgical treatment of CCA. As such, we strongly recommend a "reconsideration" of the inclusion of MRI results for LNM in the preoperative evaluation for CCA, especially when it is used as the single or principle criterion for whether or not an individual will have curative liver resection for CCA.

\section{Conclusions}

MRI has limited sensitivity and positive predictive value for the diagnosis of LNM in CCA. Therefore, surgeons should be aware of the number of false positives in determining the inoperability of CCA subjects.

\section{Additional files}

Additional file 1: Dataset 1. (CSV $16 \mathrm{~kb})$

Additional file 2: Dataset 2. (CSV $22 \mathrm{~kb}$ )

Additional file 3: Dataset 3. (CSV $52 \mathrm{~kb}$ )

\section{Abbreviations}

AJCC: American Joint Committee on Cancer; CASCAP: Cholangiocarcinoma Screening and Care Program; CCA: Cholangiocarcinoma; Cl: Confidence interval; CT: Computerized tomography; HASTE: Half-Fourier acquisition single-shot turbo spin echo; LNM: Lymph node metastasis; MRCP: Magnetic resonance cholangiopancreatography; MRI: Magnetic resonance imaging; NPV: Negative predictive values; Ov: Opisthorchis viverrini; PPV: Positive predictive values; RARE: Rapid acquisition with relaxation enhancement; US: Ultrasound

\section{Acknowledgements \\ The authors thank all the members of CASCAP, in particular the cohort members and staff from all participating institutions.}

Ethics approval and consent to participate

CASCAP was approved by Khon Kaen University Ethics Committee (HE551404) for Human Research and received written informed consent from all patient participants.

\section{Funding}

This work was supported by Khon Kaen University through the Cholangiocarcinoma Screening and Care Program (CASCAP) and the National Research Council of Thailand through the Medical Research Network of the Consortium of Thai Medical Schools.

Availability of data and materials

The datasets supporting the conclusions of this article are included within Additional files 1, 2, and 3 . 


\section{Authors' contributions}

NC, NK, BT, SK, and MS conceived, designed, and performed the research. MS wrote the analysis plan and wrote the first draft of the manuscript with the support of JT and BT. MS and NaC did the data analysis with the support of BT. $N C$, JB, and BT provided feedback of and edited the drafts of the manuscripts. All authors have seen and approved the final version of the manuscript.

\section{Consent for publication}

The patients were informed that the data concerning the case would be submitted for publication and they consented.

\section{Competing interests}

The authors declare that they have no competing interests.

\section{Publisher's Note}

Springer Nature remains neutral with regard to jurisdictional claims in published maps and institutional affiliations.

\section{Author details}

${ }^{1}$ Faculty of Public Health, Khon Kaen University, Khon Kaen, Thailand. 2Department of Radiology, Faculty of Medicine, Khon Kaen University, Khon Kaen, Thailand. ${ }^{3}$ Cholangiocarcinoma Screening and Care Program (CASCAP), Khon Kaen University, Khon Kaen, Thailand. ${ }^{4}$ Liver Fluke and Cholangiocarcinoma Research Center, Khon Kaen University, Khon Kaen, Thailand. ${ }^{5}$ Cholangiocarcinoma Center of Excellence, Faculty of Medicine, Khon Kaen University, Khon Kaen, Thailand. ${ }^{6}$ Department of Surgery, Faculty of Medicine, Khon Kaen University, Khon Kaen, Thailand. ${ }^{7}$ Department of Obstetrics and Gynecology, Faculty of Medicine, Khon Kaen University, Khon Kaen, Thailand. ${ }^{8}$ Department of Pathology, Faculty of Medicine, Khon Kaen University, Khon Kaen, Thailand. ${ }^{9}$ Data Management and Statistical Analysis Center (DAMASAC), Faculty of Public Health, Khon Kaen University, Khon Kaen, Thailand. ${ }^{10}$ Department of Microbiology, Immunology, and Tropical Medicine, School of Medicine and Health Sciences, George Washington University, Washington, USA.

\section{Received: 15 February 2017 Accepted: 11 September 2017} Published online: 20 September 2017

\section{References}

1. Khan SA, Emadossadaty S, Ladep NG, Thomas HC, Elliott P, Taylor-Robinson SD, et al. Rising trends in cholangiocarcinoma: is the ICD classification system misleading us? J Hepatol. 2012;56(4):848-54. doi:10.1016/j.jhep.2011. 11.015. PubMed PMID: 22173164

2. Green A, Uttaravichien T, Bhudhisawasdi V, Chartbanchachai W, Elkins DB, Marieng EO, et al. Cholangiocarcinoma in north east Thailand. A hospitalbased study. Trop Geogr Med. 1991;43(1-2):193-8. Epub 1991/01/01. PubMed PMID: 1661041

3. Schistosomes, liver flukes and Helicobacter pylori. IARC Working Group on the Evaluation of Carcinogenic Risks to Humans. Lyon, 7-14 June 1994. IARC Monogr Eval Carcinog Risks Hum. 1994;61:1-241. http://www.ncbi.nlm.nih. gov/pubmed/7715068

4. Sriputtha S, Khuntikeo N, Promthet S, Kamsa-Ard S. Survival rate of intrahepatic cholangiocarcinoma patients after surgical treatment in Thailand. Asian Pac J Cancer Prev. 2013;14(2):1107-10. PubMed PMID: 23621195

5. Arrington AK, Nelson RA, Falor A, Luu C, Wiatrek RL, Fakih M, et al. Impact of medical and surgical intervention on survival in patients with cholangiocarcinoma. World J Gastrointest Surg. 2013;5(6):178-86. doi:10.4240/wjgs.v5.i6.178. PubMed PMID: 23977420; PubMed Central PMCID: PMC3750129

6. Khuntikeo N, Chamadol N, Yongvanit P, Loilome W, Namwat N, Sithithaworn $\mathrm{P}$, et al. Cohort profile: cholangiocarcinoma screening and care program (CASCAP). BMC Cancer. 2015;15:459. doi:10.1186/s12885-0151475-7. PubMed PMID: 26054405; PubMed Central PMCID: PMC4459438

7. Szklaruk J, Tamm E, Charnsangavej C. Preoperative imaging of biliary tract cancers. Surg Oncol Clin N Am. 2002;11(4):865-76. PubMed PMID: 12607576

8. Manfredi R, Masselli G, Maresca G, Brizi MG, Vecchioli A, Marano P. MR imaging and MRCP of hilar cholangiocarcinoma. Abdom Imaging. 2003; 28(3):319-25. doi:10.1007/s00261-002-0047-x. PubMed PMID: 12719901

9. Jarnagin WR. Cholangiocarcinoma of the extrahepatic bile ducts. Semin Surg Oncol. 2000;19(2):156-76. PubMed PMID: 11126380
10. Pavone P, Laghi A, Passariello R. MR cholangiopancreatography in malignant biliary obstruction. Semin Ultrasound CT MR. 1999;20(5):317-23. PubMed PMID: 10527137

11. Bismuth $H$, Nakache $R$, Diamond T. Management strategies in resection for hilar cholangiocarcinoma. Ann Surg. 1992;215(1):31-8. PubMed PMID: 1309988; PubMed Central PMCID: PMC1242367

12. Lee SS, Kim MH, Lee SK, Kim TK, Seo DW, Park JS, et al. MR cholangiography versus cholangioscopy for evaluation of longitudinal extension of hilar cholangiocarcinoma. Gastrointest Endosc. 2002;56(1):25-32. PubMed PMID: 12085031

13. Lee HY, Kim SH, Lee JM, Kim SW, Jang JY, Han JK, et al. Preoperative assessment of resectability of hepatic hilar cholangiocarcinoma: combined CT and cholangiography with revised criteria. Radiology 2006;239(1):113121. doi:10.1148/radiol.2383050419. Epub 2006/02/10. PubMed PMID: 16467211

14. Bagante F, Spolverato G, Cucchetti A, Gani F, Popescu I, Ruzzenente A, et al. Defining when to offer operative treatment for intrahepatic cholangiocarcinoma: a regret-based decision curves analysis. Surgery. 2016. doi:10.1016/j.surg.2016.01.023. PubMed PMID: 27046702.

15. Weber SM, Ribero D, O'Reilly EM, Kokudo N, Miyazaki M, Pawlik TM. Intrahepatic cholangiocarcinoma: expert consensus statement. HPB. 2015; 17(8):669-80. doi:10.1111/hpb.12441. PubMed PMID: 26172134; PubMed Central PMCID: PMC4527852

16. Fisher SB, Patel SH, Kooby DA, Weber S, Bloomston M, Cho C, et al. Lymphovascular and perineural invasion as selection criteria for adjuvant therapy in intrahepatic cholangiocarcinoma: a multi-institution analysis. HPB. 2012;14(8):514-22. doi:10.1111/j.1477-2574.2012.00489.x. PubMed PMID: 22762399: PubMed Central PMCID: PMC3406348

17. Kim HJ, Kim CY, Hur YH, Koh YS, Kim JC, Kim HJ, et al. Prognostic factors for survival after curative resection of distal cholangiocarcinoma: perineural invasion and lymphovascular invasion. Surg Today. 2014;44(10):1879-86. doi:10.1007/s00595-014-0846-z. PubMed PMID: 24535697

18. Ringe Kl, Wacker F. Radiological diagnosis in cholangiocarcinoma: application of computed tomography, magnetic resonance imaging, and positron emission tomography. Best Pract Res Clin Gastroenterol. 2015;29(2): 253-65. doi:10.1016/j.bpg.2015.02.004. PubMed PMID: 25966426

19. Cho ES, Park MS, Yu JS, Kim MJ, Kim KW. Biliary ductal involvement of hilar cholangiocarcinoma: multidetector computed tomography versus magnetic resonance cholangiography. J Comput Assist Tomogr. 2007;31(1):72-8. doi:10.1097/01.rct.0000230013.24091.8e. PubMed PMID: 17259836

20. Masselli G, Manfredi R, Vecchioli A, Gualdi G. MR imaging and MR cholangiopancreatography in the preoperative evaluation of hilar cholangiocarcinoma: correlation with surgical and pathologic findings. Eur Radiol. 2008;18(10):2213-21. doi:10.1007/s00330-008-1004-z. PubMed PMID: 18463877

21. Chryssou E, Guthrie JA, Ward J, Robinson PJ. Hilar cholangiocarcinoma: MR correlation with surgical and histological findings. Clin Radiol. 2010;65(10): 781-8. doi:10.1016/j.crad.2010.04.018. PubMed PMID: 20797463

22. Pano B, Sebastia C, Ripoll E, Paredes P, Salvador R, Bunesch L, et al. Pathways of lymphatic spread in gynecologic malignancies. Radiographics. 2015;35(3):916-45. doi:10.1148/rg.2015140086. PubMed PMID: 25969940

23. Lee J SS, Stanley R, Heiken J. Computed tomography with MRI correlation. 4th ed. Philadelphia: Lippincott Willams \& Wilkins; 2006.

24. Meyers M. Dynamic radiology of the abdomen: normal and pathologic anatomy. Pathways of lymphnode metastases in cancer of the gastrointestinal and hepatobiliary tracts. 5th ed. New York: Springer-Verlag; 2000. p. 287-307.

25. Li T, Qin LX, Zhou J, Sun HC, Qiu SJ, Ye QH, et al. Staging, prognostic factors and adjuvant therapy of intrahepatic cholangiocarcinoma after curative resection. Liver Int. 2014;34(6):953-60. doi:10.1111/liv.12364. Epub 2013/10/ 19. PubMed PMID: 24134199

26. Khuntikeo N, Pugkhem A, Titapun A, Bhudhisawasdi V. Surgical management of perihilar cholangiocarcinoma: a Khon Kaen experience. J Hepatobiliary Pancreat Sci. 2014;21(8):521-4. doi:10.1002/jhbp.74. PubMed PMID: 24464976

27. Uttaravichien T, Bhudhisawasdi V, Pairojkul C, Pugkhem A. Intrahepatic cholangiocarcinoma in Thailand. J Hepato-Biliary-Pancreat Surg. 1999;6(2): 128-35. Epub 1999/07/10. PubMed PMID: 10398899

28. Woradet S, Promthet S, Songserm N, Parkin DM. Factors affecting survival time of cholangiocarcinoma patients: a prospective study in Northeast Thailand. Asian Pac J Cancer Prev 2013;14(3):1623-1627. Epub 2013/05/18. PubMed PMID: 23679246. 
29. Thunyaharn N, Promthet S, Wiangnon S, Suwanrungruang K, Kamsa-ard S. Survival of cholangiocarcinoma patients in northeastern Thailand after supportive treatment. Asian Pac J Cancer Prev. 2013;14(11):7029-32. PubMed PMID: 24377644

30. Igami T, Ebata T, Yokoyama Y, Sugawara G, Takahashi Y, Nagino M. Staging of peripheral-type intrahepatic cholangiocarcinoma: appraisal of the new TNM classification and its modifications. World J Surg. 2011;35(11):2501-9. doi:10.1007/s00268-011-1242-0. Epub 2011/09/01. PubMed PMID: 21879422

31. Ercolani G, Vetrone G, Grazi GL, Aramaki O, Cescon M, Ravaioli M, et al. Intrahepatic cholangiocarcinoma: primary liver resection and aggressive multimodal treatment of recurrence significantly prolong survival. Ann Surg. 2010;252(1):107-14. doi:10.1097/SLA.0b013e3181e462e6. Epub 2010/06/10. PubMed PMID: 20531002

32. Yedibela S, Demir R, Zhang W, Meyer T, Hohenberger W, Schonleben F. Surgical treatment of mass-forming intrahepatic cholangiocarcinoma: an 11year Western single-center experience in 107 patients. Ann Surg Oncol. 2009;16(2):404-12. doi:10.1245/s10434-008-0227-1. Epub 2008/11/28. PubMed PMID: 19037702

33. Yamashita Y, Taketomi A, Morita K, Fukuhara T, Ueda S, Sanefuji K, et al. The impact of surgical treatment and poor prognostic factors for patients with intrahepatic cholangiocarcinoma: retrospective analysis of 60 patients. Anticancer Res. 2008;28(4C):2353-9. Epub 2008/08/30. PubMed PMID: 18751418

34. Sulpice L, Rayar M, Boucher E, Pele F, Pracht M, Meunier B, et al. Intrahepatic cholangiocarcinoma: impact of genetic hemochromatosis on outcome and overall survival after surgical resection. J Surg Res. 2013;180(1):56-61. doi:10.1016/j.jss.2012.10.051. Epub 2012/11/28. PubMed PMID: 23183056

35. Hu HJ, Mao H, Shrestha A, Tan YQ, Ma WJ, Yang Q, et al. Prognostic factors and long-term outcomes of hilar cholangiocarcinoma: a single-institution experience in China. World J Gastroenterol. 2016;22(8):2601-10. doi:10.3748/wjg.v22.18.2601. PubMed PMID: 26937148; PubMed Central PMCID: PMC4768206

36. Jutric Z, Johnston WC, Hoen HM, Newell PH, Cassera MA, Hammill CW, et al. Impact of lymph node status in patients with intrahepatic cholangiocarcinoma treated by major hepatectomy: a review of the National Cancer Database. HPB. 2016;18(1):79-87. doi:10.1016/j.hpb.2015.07. 006. PubMed PMID: 26776855

37. Kim HM, Park JY, Kim KS, Park MS, Kim MJ, Park YN, et al. Intraductal ultrasonography combined with percutaneous transhepatic cholangioscopy for the preoperative evaluation of longitudinal tumor extent in hilar cholangiocarcinoma. J Gastroenterol Hepatol. 2010;25(2):286-92. doi:10.1111/j.1440-1746.2009.05944.x. PubMed PMID: 19780880

38. Adachi T, Eguchi S, Beppu T, Ueno S, Shiraishi M, Okuda K, et al. Prognostic impact of preoperative lymph node enlargement in intrahepatic cholangiocarcinoma: a multi-institutional study by the Kyushu Study Group of Liver Surgery. Ann Surg Oncol. 2015. doi:10.1245/s10434-014-4239-8. PubMed PMID: 25582737.

\section{Submit your next manuscript to BioMed Central and we will help you at every step:}

- We accept pre-submission inquiries

- Our selector tool helps you to find the most relevant journal

- We provide round the clock customer support

- Convenient online submission

- Thorough peer review

- Inclusion in PubMed and all major indexing services

- Maximum visibility for your research

Submit your manuscript at www.biomedcentral.com/submit
Biomed Central 